\title{
THE APPLICATION OF ACOUSTIC EMISSION AND ARTIFICIAL NEURAL NETWORKS IN AN ANALYSIS OF KINETICS IN THE PHASE TRANSFORMATION OF TOOL STEEL DURING AUSTEMPERING
}

\begin{abstract}
During the course of the study it involved tool steel C105U was used. The steel was austempered at temperatures of $130^{\circ} \mathrm{C}$, $160^{\circ} \mathrm{C}$ and $180^{\circ} \mathrm{C}$ respectively. Methods of acoustic emission (AE) were used to investigate the resulting effects associated with transformations and a large number of AE events were registered. Neural networks were applied to analyse these phenomena. In the tested signal, three groups of events were identified of: high, medium and low energy. The average spectral characteristics enabled the power of the signal spectrum to be determined. After completing the process, the results were compiled in the form of diagrams of the relationship of the AE incidence frequency as a function of time. Based on the results, it was found that in the austempering of tool steel, in the first stage of transformation midrib morphology is formed. Midrib is a twinned thin plate martensite. In the 2 nd stage of transformation, the intensity of the generation of medium energy events indicates the occurrence of bainite initialised by martensite. The obtained graphic of AE characteristics of tool steel austempering allow conclusions to be drawn about the kinetics and the mechanism of this transformation.
\end{abstract}

Keywords: carbon steel; austempering; lower bainite; acoustic emission (AE); neural networks

\section{Introduction}

A properly chosen method of heat treatment affects the use value of the finished materials. Well prepared products have an impact on the development of the industry, but even the slightest of defects reflects negatively on further stages of the production and products. New testing methods are in demand to enable the manufacturer to trace the kinetics of phase transformations present in processed materials during the heat treatment of them. The use of such investigation tools as acoustic emission (AE) and artificial neural networks make it possible to analyse the processes occurring in the austempering of steels.

At present, the AE method is increasingly used in different fields of science. Acoustic effects arise as a result of the dynamic trigger of accumulated energy in centres. Therefore, the source of acoustic wave formation can be the growth of dislocation annihilations, micro-cracks and the destructive processes of materials belonging to the macroscopic phenomena. In addition, a change in the status of balance in materials may cause stresses, corrosion, and radiation. AE belongs to the non-destructive test methods. Defects in materials with negligible growth, where there are no changes in stress status, do not emit any acoustic waves [1]. Metals belonging to this group of materials also emit elastic waves under certain conditions. Acoustic wave propagation in steels may be the result of dislocations, twinning, cracks and phase transformations, in particular those that occur very dynamically, such asmartensitic transformation [2].
Artificial neural networks are used primarily to resolve various problems of algorithmic approaches. In these type of models, much attention is paid to the efficiency of calculations, reducing time complexity and optimization. Due to the mentioned properties, the neural network is a very effective and reliable device for processing information and is also used in materials engineering. The use of artificial neural network enables changes of AE signal parameters originating from phase transformations to be tracked.

A another condition for creating materials with appropriate functional values is a sound knowledge of the phenomena that occur in their structures. Leading research into the effects present in steels during the breakdown of supercooled austenite was carried out by Bain and Davenport in 1930 [3] resulting in the determination of a form of diagram, $C T P_{i}$ within the temperature range $100-200^{\circ} \mathrm{C}$. In addition, they proved that the shape of diagram curve corresponds to a C-curve shape. Another scholar, Hultragen [4], stated that such a diagram structure is an effect of the building up of separate C-curves, while the occurrence of bends of diagram curves are explained as an effect of different transitions. Research carried out by Radcliffe and Rollason [5] on austempering involving measurements of changes of electrical resistance take into account the changes near point Ms. Based on the results obtained, they concluded that the swing back effect occurs near Ms temperature of eutectoid steels, while above the Ms, the swing back is characteristic of hypereutectoid steels. The swing back effect determines acceleration at the start of phase transformation [6]. Further research on pearlitic, bainitic and

$\begin{array}{ll}\text { * } & \text { INSTITUTE OF FUNDAMENTAL TECHNOLOGICAL RESEARCH, POLISH ACADEMY OF SCIENCES, 5B PAWIŃSKIEGO STR., 02-106 WARSZAWA, POLAND } \\ \text { ** } & \text { INSTITUTE OF TECHNOLOGY, KAZIMIERZ WIELKI UNIVERSITY, } 30 \text { CHODKIEWICZA STR., 85-064 BYDGOSZCZ, POLAND }\end{array}$

\# Corresponding author: gosia.lazarska@wp.pl 
martensitic transformation resulted in the obtainment of a graph with many curves and was conducted by Edwards and Kennon [7,8]. Okamoto and Oka [9,10], and T.Z.Woźniak [11], who also investigated the issue of swing back near Ms and confirmed the occurrence of this phenomenon. Bainitic transformation is still the least recognised of all phase transitions resulting in much interest being shown in it among researchers. H.K.D.H. Bhadeshia $[12,13]$ showed that the kinetics of bainitic transformation is controlled by a nucleation rate. Research related to the kinetics of bainitic transformation were also carried out by T.Z.Woźniak [14-16], who proposed a model of transformation mechanism to separate C-curves.

\section{Research methodology}

Tool steel type $\mathrm{C} 105 \mathrm{U}$ was used to conduct the research and the chemical composition analysis results for the tested material are shown in Table 1.

TABLE 1

Chemical composition of tool steel C105U (wt \%)

\begin{tabular}{|c|c|c|c|c|c|c|c|c|c|}
\hline \hline C & Mn & Si & P & S & Cr & Ni & Mo & V & Cu \\
\hline 1.09 & 0.209 & 0.37 & 0.0039 & 0.016 & 0.077 & 0.07 & 0.013 & 0.0023 & 0.132 \\
\hline
\end{tabular}

A test stand was built following framework R15 01002 in the writing of research projects introduced by the Ministry of Science and Higher Education 02, Fig. 1. The stand is used for the generation and registration of AE signals and is an innovative solution.
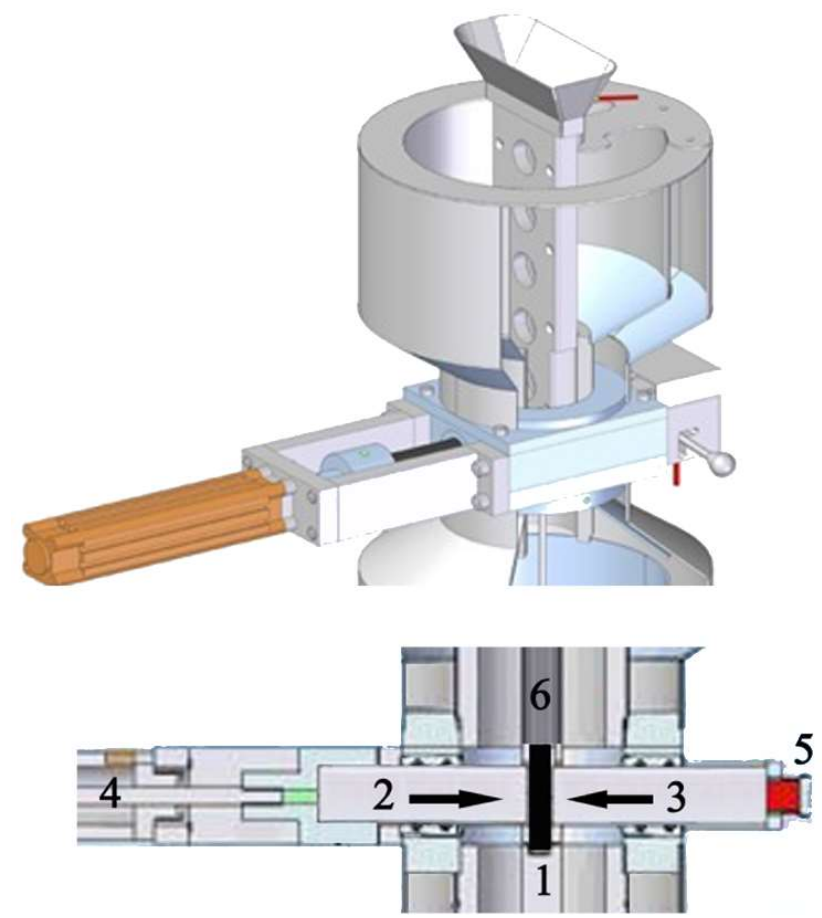

Fig. 1. The test stand in Solid Edge environment for AE tests during quenching, 1 - specimen, 2,3-waveguides that compress the specimen, 4 - pneumatic actuator, 5 - ultrasonic sensor, 6 - guide
A main element of the stand is a tank filled with oil. Inside the tank there is a guide installed for transporting specimens. For measuring temperatures, there are two thermocouples submerged in oil and integrated with a control cabinet (temperature controller) and a band heater. An ultrasonic sensor is installed to one of the mandrels to receive the AE signal generated while austempering. An air-operated actuator installed into the second mandrel enables the user to push the specimen during the test and to clamp it finally.

The $2 \mathrm{~mm}$ thick diameter specimens involved in carrying out the tests were prepared from steel supplied in the form of rolled bars. The specimens were austenitised at a temperature of $950^{\circ} \mathrm{C}$ for half an hour in a chamber oven. The austenitising conditions chosen were designed thus to dissolve a large majority of carbides. Then, the specimen was placed in the AE test stand. It was immersed in oil heated to a definite temperature. The tests were carried out in under three differing temperatures: $130^{\circ} \mathrm{C}$-near $\mathrm{Ms}, 160^{\circ} \mathrm{C}$ and $180^{\circ} \mathrm{C}$. The hold time was dependent on the occurrence of AE signals. A diagram of the heat treatment process is shown in Fig. 2.

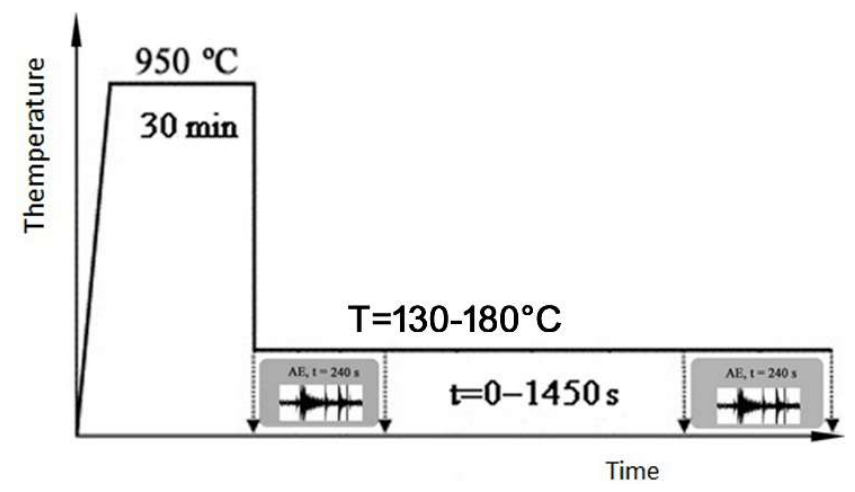

Fig. 2. Illustration of heat treatment during the experiment carried out on the stand for AE testing and the marked time periods [16]

AE signals received using the sensor were sent to the analyser where the signal was received and sent to a central unit. A scheme of the stand for AE tests is presented in Fig. 3.

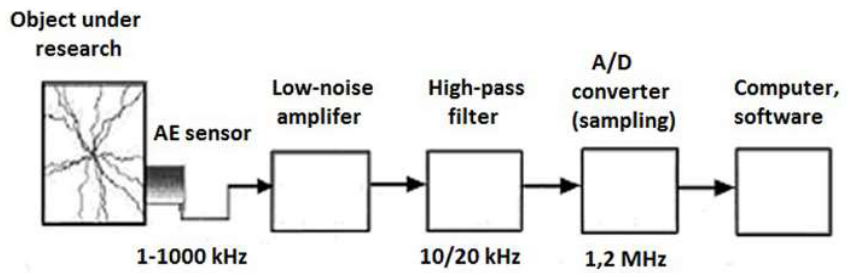

Fig. 3. Block diagram of the stand for AE tests

AE signals received using the ultrasonic sensor were sent to the analyser, received there and recorded in PC memory using A/D converters Adlink 9812. The signals in the form of elastic waves were transformed into electric signals using the sensor and the signal sampling frequency was $1.2 \mathrm{MHz}$. A band-pass filtration was applied to eliminate vibro-acoustic background witha high-pass filter with a cut-off frequency of $20 \mathrm{kHz}$ being 
used for the purpose. In addition, a low-pass filter with a cutoff frequency of $900 \mathrm{kHz}$ was employed to smooth the tested signal. The recording time signal was limited to $240 \mathrm{~s}$ due to sampling frequency and the results were plotted in the form of spectrograms. The spectrograms were developed using a window algorithm of Short Time Fourier Transform (STFT) with a Hamming window for their graphic visualisation. Each graphic box consisted of 17,640 signal samples; the spectrumcalculated from a consecutive 1647 samples located inside the box in a place where the signal achieved maximum values and which was divided into 2-kHz wide bars.

Neural networks were used to analyse AE signals generated while the tool steel was being austempered. To trace changes of AE signal, a software developed by $Z$. Ranachowski was used. The software enables the researcher to split the obtained test signal into separate segments which results in it being possible to find $\mathrm{AE}$ events within each of the separated segments and their average energy. Then, spectral characteristics were plotted for all the analysed segments. Spectral characteristics are presented as functions of frequency and represent spectral power density. The spectral characteristics make it possible to determine the power spectrum of the AE signal in the selected frequency range. The processed characteristics are vectors of patterns recognised by the artificial neural network. Afterwards, a network learning process was carried out that consisted in modifying the organisation of the developed neural network with an artificial neural network built of two layers being applied. An algorithm of "backward propagation of errors" was used to learn the vector of pattern features. The process of network learning consisted in a series of repetitions of the algorithm for all patterns successively. At the network's output, a signal was received that was dependent on the vector of features set at network input. At the final stage, a reaction of network outputs to the set patterns was tested and the relationship of $\mathrm{AE}$ occurrence frequency as a function of time was plotted.

\section{Results of the research}

An analysis of the microstructure of steel subjected to austempering at temperatures of $130^{\circ} \mathrm{C}, 160^{\circ} \mathrm{C}$ and $180^{\circ} \mathrm{C}$ was carried out. The etching of the microsection surface was performed using Vilella and Nital reagents. Details of microstructure are shown in Figs. 4-6.

Structural objects of butterfly morphology were observed in the 1st stage of the phase transition after austempering at $130^{\circ} \mathrm{C}$ and $160^{\circ} \mathrm{C}$ respectively. Their shape was similar to that observed by many previous researchers and well described by them in the literature [17]. Bainitic transformation takes place in two stages. In the first stage, a lower bainite with midrib arises. In images of microscopic examination, Figs. 4,5, it is present in the form of dark plates. The midrib is a twinned thin-plate martensite. In the second phase, a classic lower bainite occurs. Bright areas of the microscopic image indicate martensite with retained austenite (RA). In Fig. 6, lower bainite was observed

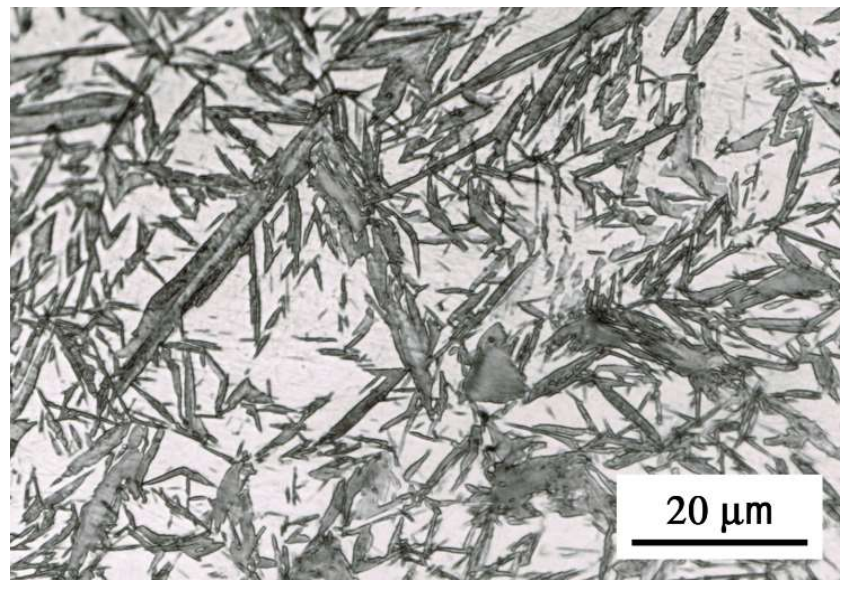

Fig. 4. Details of the microstructure of steel C105U after austempering at $130^{\circ} \mathrm{C}$, lower bainite with a midrib etched with Nital

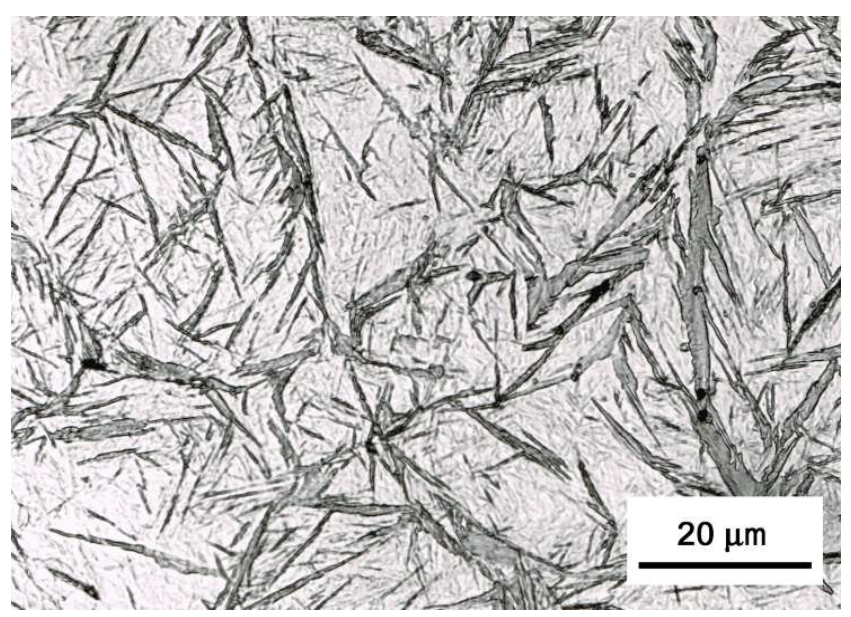

Fig. 5. Observation of microstructure details showing bainite plates with a midrib after austempering at $160^{\circ} \mathrm{C}$, etched with Vilella

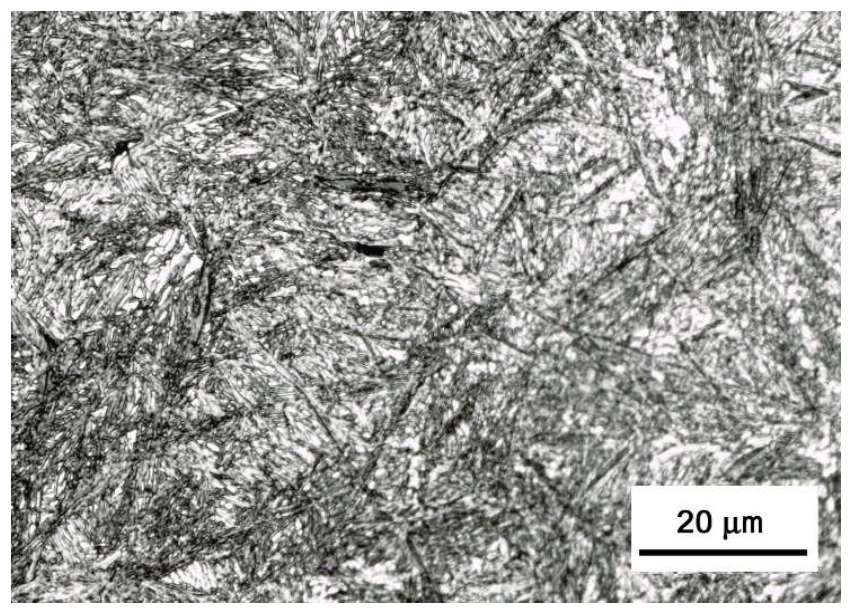

Fig. 6. Observation of microstructure details showing bainite plates during further transformation after austempering at $180^{\circ} \mathrm{C}$, etched with Nital

as the dominant component. Such morphology is characteristic for this temperature range.

AE signal spectrograms were generated for steel $\mathrm{C} 105 \mathrm{U}$ during austempering in a selected range of temperatures and are shown in Fig. 7. 
a)

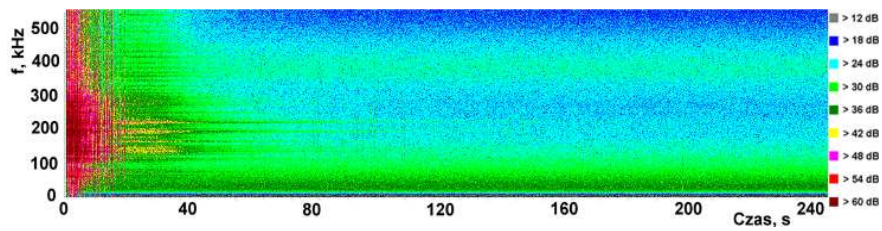

b)

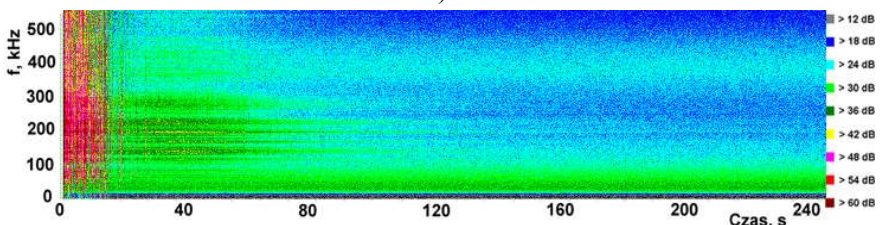

c)

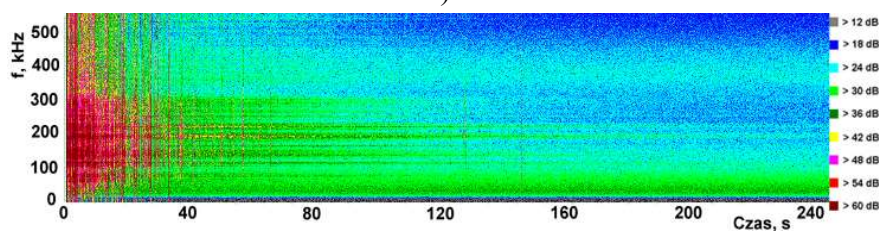

Fig. 7. EA signal power density spectrograms for steels $\mathrm{C} 105 \mathrm{U}$ in austempering at temperatures of: a) $\left.130^{\circ} \mathrm{C} \mathrm{b}\right) 160^{\circ} \mathrm{C} \mathrm{c}$ ) and $180^{\circ} \mathrm{C}$, horizontal axis-registration time, vertical axis-frequency

The spectrograms represent a spectrogram of signal power density for every moment of time of the conducted test. Differences in the intensity of the spectral band in the case of test specimens are slight. Generally, spectral components increased by a small amount at temperatures of $130^{\circ} \mathrm{C}$ and $180^{\circ} \mathrm{C}$. This was due to the fact that the first of the indicated temperatures is a temperature near Ms of the tested steel. In this temperature range, the intensity phase transition is lower. An increase in temperature induces an increase in component values of the spectrum, which is observable at $180^{\circ} \mathrm{C}$.

In a later stage, an analysis of the AE signal was undertaken using the neural network. For this purpose, the AE signal was divided into segments of time duration equal to $7.35 \mathrm{~ms}$. In these segments, AE events of different spectral characteristics and energy levels were determined. Three ranges of energy were observable by the differentiation of event energy. Events up to $10,000 \mathrm{pJ}$ were assigned to the high energy range, events $1000-5000 \mathrm{pJ}$ to the medium range and the low energy events were up to $1000 \mathrm{pJ}$. In the next step, spectral characteristics were made and are presented in Fig. 8.

Spectral power density for high energy events have considerably higher values than the densities for medium and low energy events. Events of high level energy have maximum characteristics within a range of $150-250 \mathrm{kHz}$ and spectral power densities within 40-45 dB. Medium energy events had maximum values of power density of the signal within a range of approx. 20-25 dB whereas low energy events had spectral power densities near acoustic noise.

Spectral characteristics in the form of files were used as patterns of features recognised by the artificial neural network. A process of network learning was conducted that consisted in applying a series of repetitions of the algorithm for all vectors by
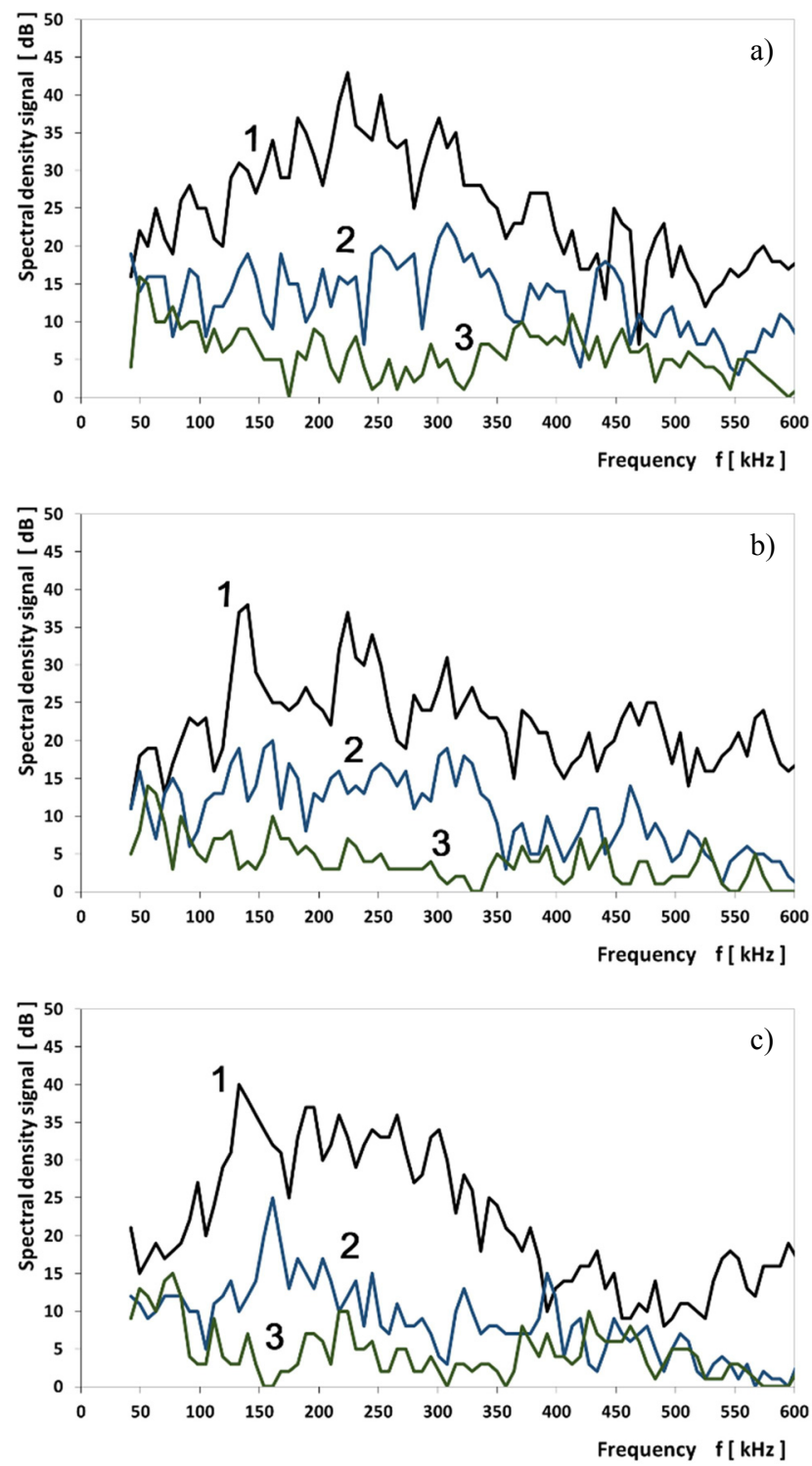

Fig. 8. Spectral characteristics for steel C105U indicating event energies of the applied segments:1-of high energy of events, 2 of medium energy events and 3 of low energy events corresponding to a) $130^{\circ} \mathrm{C}$, b) $160^{\circ} \mathrm{C}$, and c) $180^{\circ} \mathrm{C}$

using suitable learning sequences. The artificial neural network at the output generated a signal dependent on the feature vector. After the results were graphically represented, the relationships of $\mathrm{AE}$ event frequencies as a function of time are presented in Figs. 9-10.

The results of the network learning process indicate a detection of events in the signal that have spectral characteristics similar to the pattern set for the network, and thus the origin of the events from different processes. The high energy events are generated approx. 8 seconds after the start of the process. The result obtained indicates the effect of $\mathrm{AE}$ events originate from the formation of midrib that is a twinned thin plate martensite. The highest intensity of medium energy event generation in the tested process for signals obtained for steel austempering at $130^{\circ} \mathrm{C}$ was within the range $20-40$ seconds while, at $160^{\circ} \mathrm{C}$ it was within $20-50$ seconds and at $180^{\circ} \mathrm{C}$ it was between $20-50$ seconds. After that, the events decreased rapidly. The dynam- 
a)

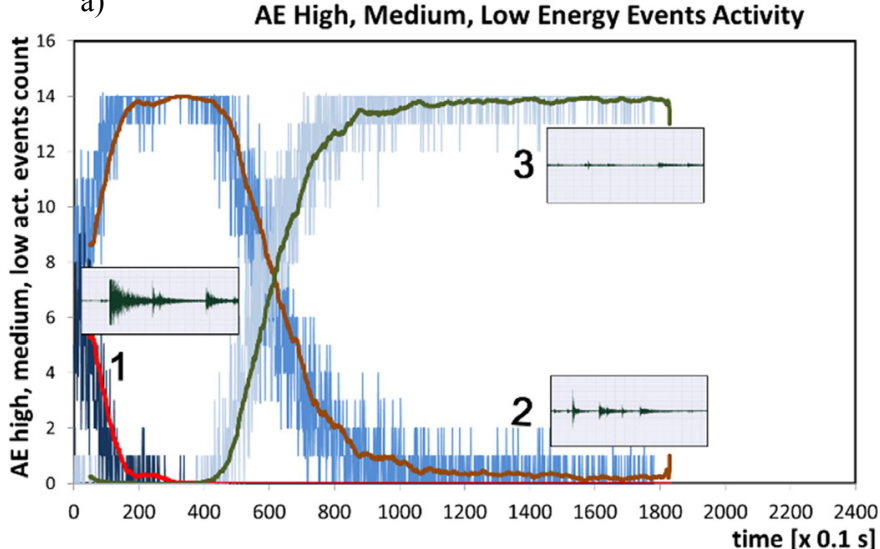

b)

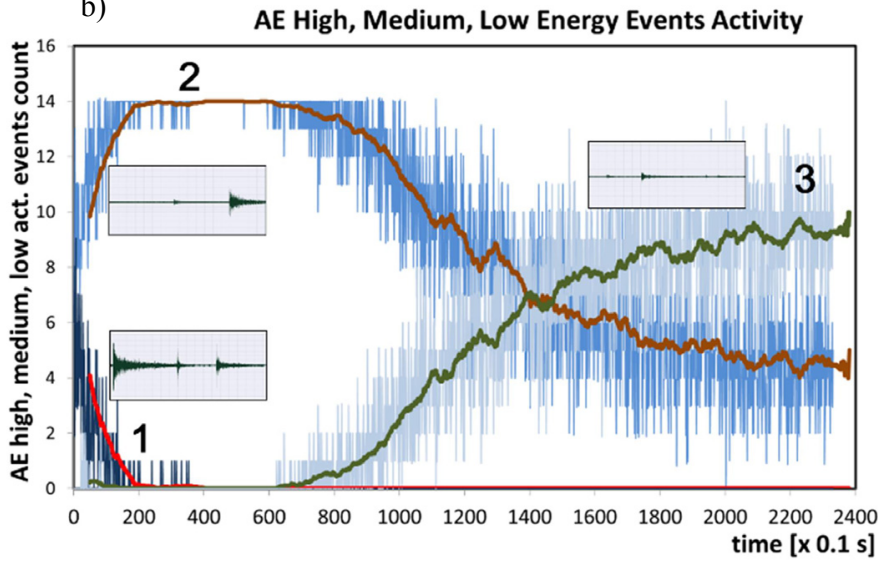

c)

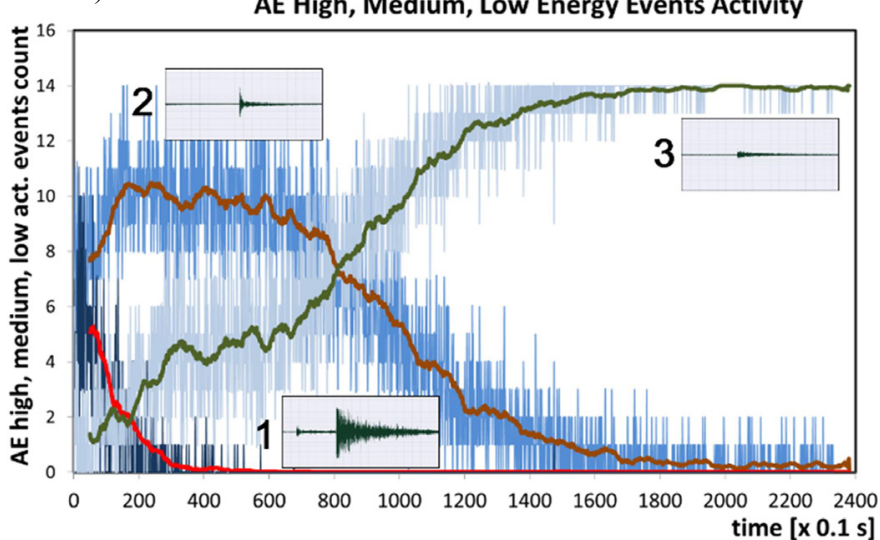

Fig. 9. The results of AE event frequency as a function of time for steel C105U, 1 - high-energetic signal standards, 2 - mid-energetic signal standards, 3 - low-energetic signal standards corresponding to a) $130^{\circ} \mathrm{C}$, b) $160^{\circ} \mathrm{C}$, and c) $180^{\circ} \mathrm{C}$

ics of the process indicates the occurrence of bainite initialised by martensite. The low energy events for the signal at $130^{\circ} \mathrm{C}$ were stabilised after approx. 100 seconds. At a temperature of $160^{\circ} \mathrm{C}$ it stabilized after approx. 20 seconds and at $180^{\circ} \mathrm{C}$ it too stabilized after approx. 20 seconds. The dynamics of events of low AE energy indicate the occurrence of bainite combined with background noise.

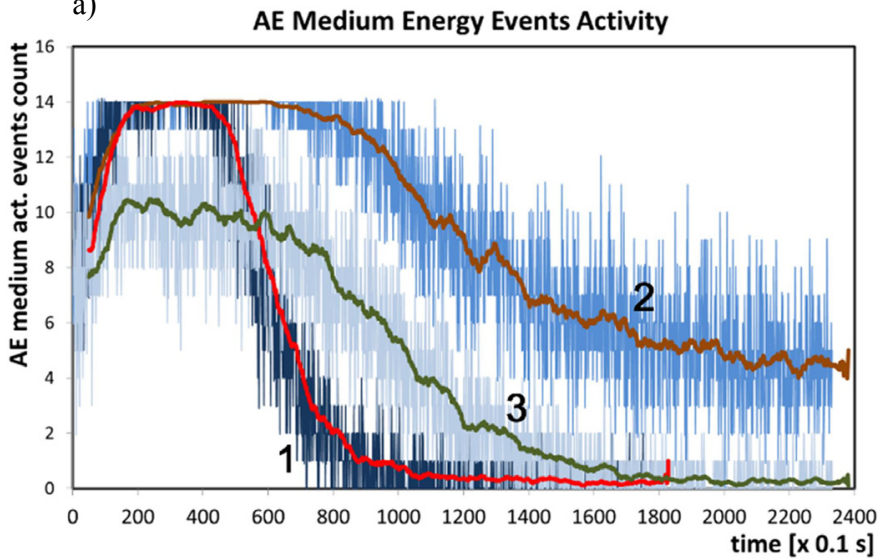

b)

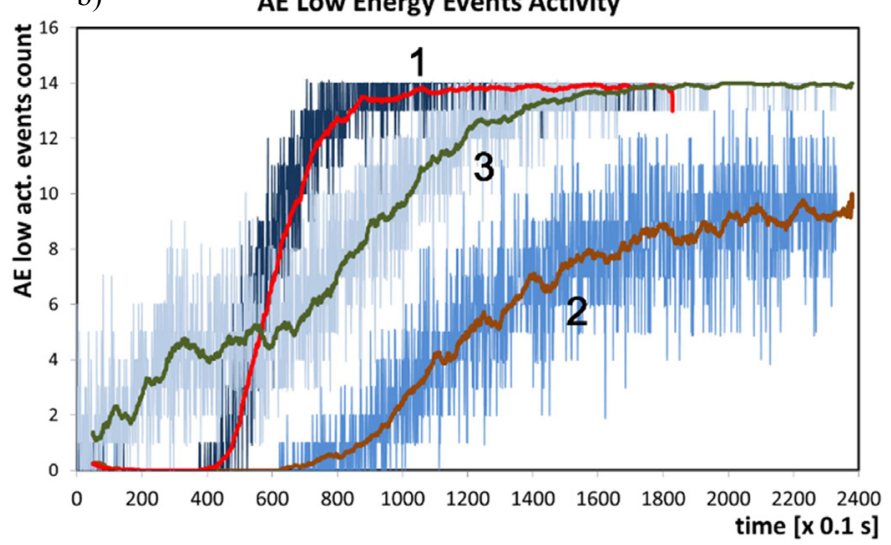

Fig. 10. The results of $\mathrm{AE}$ event frequency as a function of time for steel $\mathrm{C} 105 \mathrm{U}$ for a) medium energy events 1 at temperatures of $130^{\circ} \mathrm{C}$, $2-160^{\circ} \mathrm{C}$ and $3-180^{\circ} \mathrm{C}$ respectively, b) of low energy events $1-130^{\circ} \mathrm{C}$, $2-160^{\circ} \mathrm{C}$ and $3-180^{\circ} \mathrm{C}$

\section{Discussion}

Phase transformations as the result of heat treatment are often difficult to identify, which has an essential relationship with their volumetric participation and the overlapping of different phenomena as well. For an assessment of the merits of these effects and to identify different signal sources, acoustic emission may be used. By determining signal parameters and the frequency characteristics of waves the phenomena that occur as a result of phase transformations can be successfully controlled. Heat treatment at a low range of temperatures leads to the formation of midribs as the significant source of AE signals [18]. The application of this very sensitive method, when measuring equipment is still developing, did not allow for the detection of the signal that corresponds to the movement of dislocation groups and the effects of Frank-Red (FR) sources.FR sources are defined as sources of acoustic emission generated as a result of the annihilation processes of segments at the moment of loop closure which was proved in many studies by A. Pawełek $[19,20]$. The annihilation concerns kinks that are a part of separate segments of the same dislocation. Dislocation loops move under stresses which may induce blocking the dislocation lines in two significant points and bending the blocked dislocations. It finally 
leads to loop closure that is further expanded as an end effect. At the same time, other blocked segments of dislocation line are activated and bent which is the reason the sources that form consecutive dislocations are called Frank-Red (FR) sources. According to A. Pawełek, at the moment FR sources are activated, a cascade annihilation of bends-solitons may occur. The plastic strain in the ambient of midribs leads to the generation of FR sources that are in fact efficient sources of acoustic emission. It has also been confirmed that the number of events recorded in the AE signal over a definite time interval is proportional to the quantity and the length of dislocations that are annihilated in the same period of time. The propagation of the elastic wave is extremely fast in the entire tested object, which has an effect on the requirements that are set during the location of $\mathrm{AE}$ detectors. The concept of annihilation of dislocations has been confirmed by other studies and recognised as justified by Boiko [21]. An analysis of the events signal enables us to assess type and location of the source and seems to be a promising method to evaluate physical phenomena.

At present, the widespread and common interest of scholars in artificial neural networks derives generally from the search for methods of constructing more efficient and more reliable appliances to process the information. Based on the conducted tests, a signal of acoustic emission was obtained, in which a large amount of events were recorded. The use of neural network in this study made it possible to monitor changes in the activity of AE signal parameters. The applied software permitted separation and determination of ranges of energies of events in the recorded signal. Based on generated spectral characteristics, patterns of features were formed that are recognized by the neural network. When the network learning process was performed through the applied software it was possible to plot a relationship of events occurrence frequency as a function of time and consequently to trace the processes generated during phase transformations occurring in the tested material.

Earlier studies [22] showed that a double stage of phase transformation is present near the accelerated start of transformation. The first one leads to the formation of a lower bainite with midrib, whereas in the second stage a classic lower bainite is produced. In the microstructure of the tested steels a characteristic butterfly morphology similar in shape to the model one described in the literature was observed [17]. The microstructure of two plates, forming an obtuse angle and expanding across the grain, is also a characteristic morphology within the range of martensitic transition. In the case of bainite nucleating on midribs, the formed morphology is similar to butterfly. The effect of an accelerated start of the transformation is probably caused by a rapid generation of midrib plates [12]. Midrib initiates the start of bainitic transformation and is the first link of the formed plate of bainite. At higher temperatures, the swing back effect can be explained by the presence of lath martensite [23]. The kinetics of bainitic transformation occur in stages. In the first stage, the sub-plate nucleates on the border of austenite grain and is expanded at the same time. At the moment of the occurrence of an elastic strain inside the austenite, the growth of the sub-plate is finished. Then, there is a division of carbon to austenite phase. At the next stage, the sub-plates nucleate on top of previous sub-plates and a whole bundle is generated as a result of this [24]. New sub-plates are formed near the tops of those already existing, but at a much slower rate. The kinetics of bainitic transformation are controlled by the nucleation rate. The rate of bainite extension is much slower than martensite and the sliding movement of interface is slower, too. The reason behind these differences may be plastic deformation that occurs during bainite growth.

\section{Conclusions}

The development of the industry demands higher requirements for the produced materials and developing technology creates better and better capabilities for testing using state of the art research techniques. As a result of the tests carried out using acoustic emission and the artificial neural network, an analysis of the phenomena occurring while steel $\mathrm{C} 105 \mathrm{U}$ was austempered at temperatures of $130^{\circ} \mathrm{C}, 160^{\circ} \mathrm{C}$ and $180^{\circ} \mathrm{C}$, was undertaken.

1. In the recorded signal, events of different energies and spectral characteristics were detected. A successful attempt was undertaken to determine energy ranges based on the differentiated energy of the events which occurred.

2. The results generated from the network learning process indicate the occurrence of events in the AE signal that have spectral characteristics similar to patterns set for the network. It indicates that the events originate from different processes.

3. Events of high energy indicate that they originate from the growing midrib. Events of medium energy show the detection of bainite initialised by martensite whereas hereas the dynamics of events of low energy show the detection of bainite combined with background noise.

4. It can be concluded that the signals acoustic emission make it possible to identify the phenomena of an e accelerated start of phase transformation.

5. The methods of acoustic emission and artificial neural networks used in this research can be applied to studies of the kinetics of phase transformations that occur in steels.

\section{REFERENCES}

[1] I. Malecki, J. Ranachowski, Emisja Akustyczna. Źródła Metody Zastosowania, Wyd. Pascal 1994, Warszawa.

[2] T.Z. Wozniak, Materials Characterization 59, 708-16 (2008).

[3] E.S. Davenport, E.C. Bain, Transformation of Austenite at Subcritical Temperatures. Trans. AIME 90 (1930).

[4] A. Hultgren, Trans. ASM 39, 915 (1947).

[5] S.V. Radcliffe, E.C. Rollason, JISI 191, 56, (1959).

[6] Mokuang Kang, Ming-Xing Zhang, Ming Zhu, Acta Mater. 54, 2121-2129 (2006).

[7] N.F. Kennon, Met. Trans. 9, 57 (1978). 
[8] N.F. Kennon, N.A. Kaye, Met. Trans. 13, 975 (1982).

[9] M. Oka, H. Okamoto, Met. Trans. 19, 447 (1988).

[10] H. Okamoto, M. Oka, Met. Trans. 17, 1113 (1986).

[11] T.Z. Wozniak, K. Różniatowski, Z. Ranachowski, Metals and Materials International 17, 365 (2011).

[12] H.K.D.H. Bhadeshia and C. H. Young, Mater. Sci. Tech. 10, 209 (1994).

[13] H.K.D.H. Bhadeshia, Bainite in Steels, 2-nd ed. The Institute of Materials, London (2001).

[14] T.Z. Wozniak, Materials Science and Engineering A408, 309 (2005).

[15] T.Z. Wozniak, K. Różniatowski, Z. Ranachowski, Kovove Materialy-Metallic Materials 49, 319 (2011).

[16] T.Z. Wozniak, Z.Ranachowski, P. Ranachowski, W. Ozgowicz, A. Trafarski, Arch. Met. Mater. 59, 1705-1712 (2014).
[17] Umemoto M., Hyodo T., Maeda T., Tamura I., Acta Metall. 32, 1191-1203 (1984).

[18] T.Z. Wozniak, Materials Characterization 59(6), 708 (2008).

[19] A. Pawełek, J. Kuśnierz, J. Bogucka, J. Jasiński, Z. Ranachowski, Arch. of Metall. and Mater. 53 (2008).

[20] A. Pawełek, J. Kuśnierz, J. Bogucka, J. Jasiński, Z. Ranachowski, P. Ranachowski, F. Rajmund, T. Dębowski, Arch. of Acoust. 32, 955-962 (1997).

[21] V.S. Boiko, Phys. Stat. Sol.(b) 55, 477 (1973).

[22] T.Z. Wozniak, J. Jeleńkowski, Zesz. Nauk. 193, Mech. 38, ATR, Bydgoszcz, 103 (1995).

[23] G.Spanos, H.S.Fang, H.I.Aaronson:Met. Trans. 21, 1381 (1990).

[24] G.I. Rees, H.K.D.H. Bhadeshia, Mater. Sci. Technol. 8, 985-993 (1992). 